\section{Modeling the dynamics of HCV}

\section{By Tracey Baas, Senior Editor}

Los Alamos National Laboratory researchers have developed a computational model that describes the dynamics of antiviral resistance in HCV patients. ${ }^{1}$ The model could help scientists not only design better clinical trials using the best combinations of candidate drugs but also move away from including the standard of care in each trial.

The current standard of care for chronic $\mathrm{HCV}$ is pegylated interferon- $\alpha$ (IFNA; IFN- $\alpha$ ) and ribavirin, two nonspecific antivirals. However, the treatment is only effective in just over $50 \%$ of patients, and side effects include flu-like illness, fever and fatigue.

The quest for more efficacious therapeutics has focused on compounds that target specific viral components. There are 2 compounds in Phase III, 11 in Phase II and 4 in Phase I trials (see Table 1 in Baas, T., SciBX 3(5); doi:10.1038/scibx.2010.138).

All are vying to become the first new HCV treatments approved in a decade, the most advanced of which is telaprevir, an HCV nonstructural protein 3 (NS3) and NS4A serine protease inhibitor from Vertex Pharmaceuticals Inc.

However, drug-resistant variants of $\mathrm{HCV}$ emerge at frequencies of about $5 \%-20 \%$ of the total virus population as early as the second day after monotherapy with telaprevir, making HCV the virus with the most rapidly developing drug resistance ever observed. ${ }^{2}$

Alan Perelson, a research scientist at Los Alamos, and his team set out to determine whether viral resistance and population dynamics could be modeled as a first step toward predicting therapeutic outcome and the subsequent design of optimized treatments.

As a starting point, the team used a data set from HCV patients who received either telaprevir monotherapy or telaprevir in combination with pegylated IFN- $\alpha$.

First, the team showed that the high rates of mutation and early emergence of resistance imply the pre-existence of drug-resistant variants. Once monotherapy starts, and depending on their fitness, these variants out-compete wild-type virus.

Next, the researchers modeled viral dynamics during a 14-day course of telaprevir monotherapy and the two-drug combination. The model fit the growth data seen in patients for both the resistant variants and the wild-type virus.

For patients treated with a combination of telaprevir and pegylated
IFN- $\alpha$, the model could not distinguish the individual drug efficacies of telaprevir and IFN- $\alpha$ because they both act as antiviral agents. However, it could show that telaprevir-resistant virus was sensitive to pegylated IFN- $\alpha$, supporting use of the combination.

According to Perelson, although the work uses telaprevir data, monotherapy with any protease inhibitor or non-nucleoside polymerase inhibitor would lead to a similarly rapid appearance of $\mathrm{HCV}$ drug resistance.

"Because both protease and polymerase inhibitors act to suppress viral replication, the model also allows us to study drug resistance during treatment with polymerase inhibitors," Perelson told SciBX.

However, evaluating inhibitors that target $\mathrm{HCV}$ proteins lacking a known function, such as NS5A, could prove more challenging.

A recent paper from Bristol-Myers Squibb Co. ${ }^{3}$ did indeed show that an "NS5A inhibitor gave rise to rapid viral load declines, similar to what has been seen with protease inhibitors," Perelson said. "Our model used in conjunction with detailed experimental data, when publicly available, could also help us understand the mechanisms of NS5A inhibitors."

The team included researchers from Los Alamos, Oakland University and the University of Illinois at Chicago. The work was published in Science Translational Medicine.

\section{Beyond monotherapy}

Although modeling monotherapies provides valuable insights into viral dynamics, the challenge lies in how to use this information to design the most efficient combination therapies.

"Monotherapy modeling provides the necessary data and lays the groundwork for future predictive models, which will one day provide a framework for rational therapy strategies," noted Robert Kauffman, SVP of clinical development and CMO at Vertex. "Ultimately it is the hope that these models will predict the best combination of candidate drugs so that clinical trials can move away from including the standard of care in each trial."

He added: "What one really needs is a model to evaluate triple therapies-ultimately to help researchers design better clinical studies."

But he noted that work such as that of Perelson's group "is necessary before good predictive modeling can be done for combination therapies," because fewer HCV escape mutants emerge during combination therapy. This limits the amount of viral load and viral variant data available to build predictive models. Monotherapy analyses are not constrained by this.

Vertex says it has already begun to work on the more complex models.

"Vertex has an in-house modeling group that has already evaluated the triple therapy used in our successful PROVE 3 trial," Kauffman told SciBX. "They used the viral load data to develop a model which paralleled the clinical outcome, and we have used that information to design our future clinical studies." 


\section{ANALYSIS}

In PROVE 3, 52\% of patients treated with a combination of telaprevir, pegylated IFN- $\alpha$ and ribavirin experienced a sustained responsewith no signs of $\mathrm{HCV}$ - compared with $14 \%$ of those given pegylated IFN- $\alpha$ and ribavirin alone. ${ }^{4}$ Patients in the study had previously failed to respond to the two-drug combination or had relapsed.

Vertex and partner Janssen Pharmaceutica N.V., a unit of Johnson \& Johnson, will now evaluate the three-compound cocktail from PROVE 3 in naïve patients and a more well-characterized group of patients showing no response to pegylated IFN- $\alpha$ and ribavirin.

Merck \& Co. Inc. is also conducting a Phase III trial of a combination of boceprevir, pegylated IFN- $\alpha$ and ribavirin in naïve patients and in nonresponders to pegylated IFN- $\alpha$ and ribavirin.

Bristol-Myers has started a Phase IIa study of two unapproved compounds-the NS5A inhibitor BMS-790052 and the NS3 inhibitor BMS-650032-with and without pegylated IFN- $\alpha$ and ribavirin. Results are expected in $2 \mathrm{H} 10$.

Perelson's team is now developing models that include multiple emerging viral variants. This strategy will provide a more accurate description and prediction of the dynamics of different drug-resistant variants during therapy. Such viral dynamic information coupled with detailed information about pre-existing HCV variants in a patient could help establish a framework for matching drugs with specific drug-resistance profiles.

Perelson said the computational method has not been patented.

Baas, T. SciBX 3(20); doi:10.1038/scibx.2010.608

Published online May 20, 2010

\section{REFERENCES}

1. Rong, L. et al. Sci. Transl. Med.; published online May 5, 2010; doi:10.1126/scitranslmed.3000544

Contact: Alan S. Perelson, Los Alamos National Laboratory, Los Alamos, N.M. e-mail: asp@lanl.gov

2. Kieffer, T. et al. Hepatology 46, 631-639 (2007)

3. Gao, M. et al. Nature 465, 96-100 (2010)

4. McHutchison, J.G. et al. N. Engl. J. Med. 362, 1292-1303 (2010)

COMPANIES AND INSTITUTIONS MENTIONED

Bristol-Myers Squibb Co. (NYSE:BMY), New York, N.Y. Johnson \& Johnson (NYSE:JNJ), New Brunswick, N.J.

Los Alamos National Laboratory, Los Alamos, N.M.

Merck \& Co. Inc. (NYSE:MRK), Whitehouse Station, N.J.

Oakland University, Rochester, Mich.

University of Illinois at Chicago, Chicago, III.

Vertex Pharmaceuticals Inc. (NASDAQ:VRTX), Cambridge, Mass. 